QUIPURAMAYOC $\mid$ Revista de la Facultad de Ciencias Contables

Vol. 20 N. 37 pp. 26-39 (2012) UNMSM, Lima, Perú

ISSN: 1560-9103 (versión impresa) / ISSN: 1609-8196 (versión electrónica)

\title{
APLICACIÓN DE LA NIC 19, BENEFICIOS A LOS EMPLEADOS, Y PARTICIPACIÓN DE UTILIDADES EN EL PERÚ
}

\author{
APPLICATION OF IAS 19 EMPLOYEE BENEFITS - PARTICIPATION OF \\ PROFITS IN PERU
}

\section{Agustín Rodríguez Chávez}

Docente Asociado de la Facultad de Ciencias Contables - UNMSM

[Recepción: Julio de 2012/ Conformidad: Agosto 2012]

\section{RESUMEN}

En enero del año 2011, el Consejo Normativo de Contabilidad resuelve precisar que el reconocimiento de las participaciones de los trabajadores en las utilidades determinadas sobre bases tributarias deberá hacerse de acuerdo con la NIC 19, Beneficios a los Empleados, y no por analogía con la NIC 12, Impuesto a las Ganancias, o la NIC 37, Provisiones, Pasivos Contingentes y Activos Contingentes.

Esto en base a consultas efectuadas al Comité de Interpretaciones de las Normas Internacionales de Información Financiera (CINIIF), quien en su sesión del 04 de noviembre de 2010 concluye, respecto a la contabilización de la participación de los trabajadores en las utilidades calculada de conformidad con la legislación fiscal, que el reconocimiento de la participación de los trabajadores se realizará de acuerdo a lo establecido en la Norma Internacional de Contabilidad NIC 19, Beneficios a los Empleados, sólo por los gastos de compensación por los servicios prestados en el ejercicio; en consecuencia no se registra un activo diferido o un pasivo diferido requerido en la NIC 12. Impuesto a las Ganancias.

Esta disposición obliga a las empresas a efectuar cambios en sus prácticas y políticas contables para el reconocimiento de la participación de utilidades y su cumplimiento con lo dispuesto en dicha norma. Sin embargo,

\begin{abstract}
In January 2011, the Accounting Standards Board (Consejo Normativo de Contabilidad) recognized profit sharing of employees on tax bases in accordance with IAS 19 Employee Benefits and not by analogy with IAS12 - Tax Income or IAS 37 Provisions, Contingent Liabilities and Contingent Assets.

This is based on consultations made to the International Financial Reporting Interpretation Committee - IFRIC, which at its meeting on November 4, 2010, concluded, in respect to the accounting of profit-sharing of employees, which was calculated in accordance with tax legislation, that the recognition of the profitsharing of employees will be in accordance with the provision of the International Accounting Standard 19, Employee's Benefits, and this is only for the compensation of services provided during the year. Therefore, not should be recorded a deferred tax assets or a deferred tax liability as is required in IAS 12, Income Taxes.

This legal disposition requires companies to make changes in its account practices and policies in order to recognize the profit sharing and compliance on the provisions of this rule. However, there is a lack of precision on the effects of management, a lack of information on compliance with tax standards and a lack of analysis on the formulation of financial statements.
\end{abstract}

As a result of this, costing processes, accoun-

* Contador Público Colegiado Certificado. Maestrante en Costos y Presupuestos. Doctorante en Ciencias Empresariales. E-mail: arodriguezc@unmsm.edu.pe. 
les falta precisión o un análisis profundo de los efectos en la gestión y cumplimiento de las normas tributarias y la formulación de los estados financieros.

Producto de ello se ven afectados los procesos de costeo, registros contables, determinación de los resultados del ejercicio, y las mediciones financieras por cambio en la estructura de los estados financieros.

Hemos apreciado que al cierre del ejercicio 2011 y los meses siguientes muchas empresas no han cumplido con la aplicación de la norma y su precisión en nuestro país o lo han cumplido parcialmente; por lo que es nuestro propósito efectuar el análisis necesario, para exponer las razones de incumplimiento y presentar una propuesta de prácticas y políticas para su mejor adecuación. Pretendemos que este aporte permita una mejor comprensión de los alcances de dicha norma en nuestro país y que la comunidad empresarial y contable cuente con una opción de aplicación

Palabras Clave: NIC 19, participación de utilidades, costos de mano de obra, estructura de costos, estructura financiera, prácticas y políticas contables.

\section{INTRODUCCIÓN}

Cuando en los años setenta del siglo pasado se estableció la participación de utilidades de los trabajadores tenía el propósito de reconocer en el capital de la empresa el aporte del capital humano como una parte de los resultados del esfuerzo de su labor. En el transcurso de los años, los modelos de cálculo y distribución no han cambiado sustantivamente: determinación sobre base tributaria, distribución sobre la base de Remuneraciones y días laborados. En esos tiempos no se discutía sobre el reconocimiento de dichas participaciones y se reconocían como parte del resultado de gestión (estado de resultados) después de los resultados operativos y antes del impuesto a la renta. ting records, determination of profits or loss and financial measurements will be affected by the changes in the structure of the financial statements.

We have seen at year end of 2011 and on the following months that many companies have failed in the implementation of the standard or have only partially fulfilled it, and it's our aim to make the necessary analysis, and to explain the reason for this breach and submit a proposal for practices and policies for compliance. We hope that this contribution allows a better understanding of the scope of this rule in our country and that business community and accounting count with an option of application.

Keywords: Internal Control, Agencies of the State, Government Sector

Los cambios ocurridos en los ochentas fueron modificando gradualmente el reconocimiento de este hecho económico, hasta finalmente considerarlo como un beneficio de los trabajadores y por consiguiente, tal como propone la norma, esten asociados a los costos de la mano de obra.

Esto si cambia sustantivamente las estructuras de la determinación de los resultados de gestión y el estado de situación de la empresa, por ende impacta en las formas de medición económicas financieras y muchas bases para la determinación de cumplimientos de obligaciones tributarias formales y sustantivas. Aún se está discutiendo si el cambio redunda en la determinación del monto de participaciones. 
Con un análisis en cada uno de estos aspectos expondremos el impacto en los aspectos financieros, económicos tributarios, laboral, de aplicación de las NIC's, costos.

\begin{tabular}{|c|c|c|}
\hline & \multicolumn{2}{|c|}{ EMPRESAS EN BOLSA } \\
\hline ESTADO DE SITUACION FINANCIERA & INTRADEVCO & GOODYEAR \\
\hline \multicolumn{3}{|l|}{ AVTIVO } \\
\hline \multicolumn{3}{|l|}{ Activo corriente } \\
\hline \multicolumn{3}{|l|}{ Total activo no corriente } \\
\hline TOTAL ACTIVO & 555,703 & 472,940 \\
\hline \multicolumn{3}{|l|}{ PASIVO Y PATRIMONIO NETO } \\
\hline \multirow{4}{*}{$\begin{array}{l}\text { Pasivo corriente } \\
\text { Total pasivo no corriente } \\
\text { Patrimonio neto } \\
\text { TOTAL PASIVO Y PATRIMONIO NETO }\end{array}$} & 200,732 & 79,939 \\
\hline & 68,942 & 30,081 \\
\hline & 286,029 & 362,920 \\
\hline & 555,703 & 472,940 \\
\hline Existencias & 143,871 & 39,721 \\
\hline \multicolumn{3}{|l|}{ ESTADO DE RESULTADOS } \\
\hline Ventas netas & 380,418 & 455,829 \\
\hline Costo de ventas & $(284,224)$ & $(368,891)$ \\
\hline Utilidad bruta & 96,194 & 86,938 \\
\hline Gastos de operación- neto & $(75,858)$ & $(42,779)$ \\
\hline Utilidad de operación & 20,336 & 44,159 \\
\hline \multirow{3}{*}{$\begin{array}{l}\text { Otros ingresos y gastos: } \\
\text { Utilidad (Pérdida) antes Partic. e Imp }\end{array}$} & $-6,224$ & 1,640 \\
\hline & 14,112 & 45,799 \\
\hline & - & - \\
\hline \multirow{2}{*}{$\begin{array}{l}\text { Impuesto a la Renta } \\
\text { Resultado del Ejercicio }\end{array}$} & $(4,293)$ & $(14,955)$ \\
\hline & 9,819 & 30,844 \\
\hline
\end{tabular}

Podemos ver que las empresas registradas en bolsa aplican la distribución de las participaciones entre los costos y gastos. Sin embargo, cuando analizamos los resultados presentados en el ejercicio 2010 (incluyendo en el resultado la partida de participaciones de trabajadores) reformulados para su presentación conjuntamente con el ejercicio 2011, apreciamos que la distribución se efectúa entre el costo de ventas y los gastos operativos y no se asigna a los costos de los inventarios de productos terminados $\mathrm{y}$ en proceso que sin duda tienen costo de mano de obra.

Por ejemplo, en los estados financieros de Goodyear en los EEF publicados en la WEB de la Bolsa de Valores de Lima se incluye : "De acuerdo con PCGA, en Perú la compañía mostró el monto de la participación de los trabajadores en las utilidades como gasto en la línea inmediatamente anterior a la de impuesto a la renta. De acuerdo con la NIC 19 Beneficios a los empleados, la participación de los trabajadores en las utilidades corresponde a un beneficio de los trabajadores relacionado directamente con la prestación de sus servicios a la entidad. En consecuencia, el cargo de esta partida debe afectar las categorías de gastos a las que corresponde la función del trabajador beneficiario de la participación. De esta forma el cargo total por el beneficio se debe asignar entre, el costo de ventas, los gastos de administración y de distribución y los gastos de venta. El importe de las participaciones de los trabajadores que ascendió a s/.6,338,000 fue reclasificado al costo de ventas, $\mathrm{s} / .4,893,000$, gasto de venta $\mathrm{s} / .719,000$, y gasto de administración y de distribución, s/.726,000.

\begin{tabular}{|c|c|c|c|c|}
\hline \multirow[b]{2}{*}{ ESTADO DE SITUACION FINANCIERA } & \multicolumn{2}{|c|}{ INTRADVECO EEFF2010 } & \multicolumn{2}{|c|}{ GOODYEAR EEFF 2010} \\
\hline & \begin{tabular}{|l|} 
EN 2011 \\
\end{tabular} & EN 2010 & \begin{tabular}{|l|} 
EN 2011 \\
\end{tabular} & EN 2010 \\
\hline \multicolumn{5}{|l|}{\begin{tabular}{|l|} 
AVTIVO \\
\end{tabular}} \\
\hline \multicolumn{5}{|l|}{ Activo corriente } \\
\hline \multirow{2}{*}{\multicolumn{5}{|c|}{$\begin{array}{l}\text { Total activo no corriente } \\
\text { TOTAL ACTIVO }\end{array}$}} \\
\hline & & & & \\
\hline \multicolumn{5}{|l|}{ PASIVO Y PATRIMONIO NETO } \\
\hline \multirow{4}{*}{$\begin{array}{l}\text { Pasivo corriente } \\
\text { Total pasivo no corriente } \\
\text { Patrimonio neto } \\
\text { TOTAL PASIVO Y PATRIMONIO NETO } \\
\end{array}$} & 193,039 & 193,039 & 76,952 & 76,952 \\
\hline & 50,409 & 21,910 & 30,166 & \\
\hline & 273,560 & 183,582 & 342,797 & 272,487 \\
\hline & 517,008 & 398,531 & 449,915 & 349,439 \\
\hline Existencias & 139,742 & 142,124 & 30,214 & 30,214 \\
\hline \multicolumn{5}{|l|}{ ESTADO DE RESULTADOS } \\
\hline \multicolumn{5}{|l|}{ Ventas netas } \\
\hline \multirow{2}{*}{$\begin{array}{l}\text { Costo de ventas } \\
\text { Utilidad bruta }\end{array}$} & $(266,218)$ & $(256,055)$ & $(308,959)$ & $(303,944)$ \\
\hline & 109,909 & 120,072 & 91,514 & 96,529 \\
\hline \multirow{2}{*}{$\begin{array}{l}\text { Gastos de operación- neto } \\
\text { Utilidad de operación }\end{array}$} & $(67,001)$ & $(62,001)$ & $(39,232)$ & $(37,787)$ \\
\hline & 42,908 & 58,071 & 52,282 & 58,742 \\
\hline \multirow{2}{*}{$\begin{array}{l}\text { Otros ingresos y gastos: } \\
\text { Utilidad (Pérdida) antes Partic. e Imp }\end{array}$} & $-8,309$ & $-8,310$ & 3,039 & 3,039 \\
\hline & 34,599 & 49,761 & 55,321 & 61,781 \\
\hline \multirow{3}{*}{$\begin{array}{l}\text { Participación de utilidades } \\
\text { Impuesto a la Renta } \\
\text { Resultado del Ejercicio }\end{array}$} & & $(4,977)$ & - & $(6,371)$ \\
\hline & $(10,196)$ & $(13,437)$ & $(17,179)$ & $(17,206)$ \\
\hline & 24,403 & 31,347 & 38,142 & 38,204 \\
\hline
\end{tabular}

Por otro lado, existen empresas que incluso en el ejercicio 2011 no han efectuado la distribución de las participaciones, consideramos que en muchos casos es por desconocimiento o falta de modelos para su aplicación. Esperamos contribuir a que las empresas puedan establecer sus estructuras financieras de acuerdo a como el mundo globalizado y altamente competitivo. 
Las gerencias tendrían una estructura de costos que les permita medir los resultados de su gestión con indicadores más precisos.

\begin{tabular}{|c|c|c|c|c|}
\hline \multicolumn{5}{|c|}{ EMPRESAS FUERA BOLSA } \\
\hline \multirow{2}{*}{\begin{tabular}{|l|} 
ESTADO DE SITUACION FI \\
AVTIVO
\end{tabular}} & \multicolumn{2}{|c|}{ TRANSPORTE } & \multicolumn{2}{|c|}{ ELECTRODOMESTICO } \\
\hline & & & & \\
\hline Activo corriente & 14,254 & & 6,550 & \\
\hline Total activo no corriente & 33,365 & & 3,650 & \\
\hline TOTAL ACTIVO & 47,619 & & 10,200 & \\
\hline \multicolumn{2}{|c|}{ PASIVO Y PATRIMONIO NETO } & & & \\
\hline \multirow{4}{*}{$\begin{array}{l}\text { Pasivo corriente } \\
\text { Total pasivo no corriente } \\
\text { Patrimonio neto } \\
\text { TOTAL PASIVO Y PATRIM }\end{array}$} & 14,109 & & 2,600 & \\
\hline & 6,375 & & 300 & \\
\hline & 27,135 & & 7,300 & \\
\hline & 47,619 & & 10,200 & \\
\hline Existencias & 851 & & $1 \mathrm{D0106}$ & \\
\hline \multicolumn{5}{|l|}{ ESTADO DE RESULTADOS } \\
\hline \multirow{5}{*}{$\begin{array}{l}\text { Ventas netas } \\
\text { Costo de ventas } \\
\text { Utilidad bruta } \\
\text { Gastos de operación- neto } \\
\text { Utilidad de operación }\end{array}$} & 114,955 & $100 \%$ & 6,380 & $100 \%$ \\
\hline & $(104,028)$ & $-90 \%$ & $(3,650)$ & $-57 \%$ \\
\hline & 10,927 & $10 \%$ & 2,730 & $43 \%$ \\
\hline & $(6,188)$ & $-5 \%$ & $(750)$ & $-12 \%$ \\
\hline & 4,739 & $4 \%$ & 1,980 & $31 \%$ \\
\hline \multirow{2}{*}{$\begin{array}{l}\text { Otros ingresos y gastos: } \\
\text { Utilidad (Pérdida) antes } \mathrm{Pa}\end{array}$} & 657 & $1 \%$ & 250 & $4 \%$ \\
\hline & 5,396 & $5 \%$ & 2,230 & $35 \%$ \\
\hline \multirow{3}{*}{$\begin{array}{l}\text { Participación de utilidade } \\
\text { Impuesto a la Renta } \\
\text { Resultado del Eiercicio }\end{array}$} & $(331)$ & $0 \%$ & $(112)$ & $-2 \%$ \\
\hline & $(1,888)$ & $-2 \%$ & $(651)$ & $-10 \%$ \\
\hline & 3,508 & $3 \%$ & 1,468 & $23 \%$ \\
\hline
\end{tabular}

ALCANCE DE LA NIC19 BENEFICIOS A LOS EMPLEADOS CON RESPECTO A LA PARTICIPACIÓN DE LOS TRABAJADORES EN LAS UTILIDADES

\section{DE LA EMPRESA}

El objetivo de la NIC 19 es señalar el tratamiento contable y la información a revelar respecto de los beneficios de los empleados; dentro de estos beneficios está considerada la participación de los trabajadores en las utilidades de la empresa.

\section{Alcance}

Esta norma debe ser aplicada por los empleadores al contabilizar todas las retribuciones de los empleados que procedan de:

(a) acuerdos formales celebrados entre una entidad y sus empleados; (b)requerimientos legales o acuerdos tomados en determinados sectores; (c)prácticas no formalizadas que generan obligaciones implícitas.
La participación de los trabajadores en las utilidades de la empresa se encontraría contemplada en el punto b) del alcance de la norma.

\section{Beneficios a los empleados}

La NIC 19 clasifica los beneficios a los empleados en cuatro categorías:

(a) beneficios a corto plazo, tales como sueldos, salarios y contribuciones a la seguridad social, ausencias remuneradas por enfermedad y por otros motivos; participación en las utilidades e incentivos (si se pagan dentro de los doce meses siguientes tras el cierre del periodo); y beneficios no monetarios (tales como asistencia médica, alojamiento, automóviles y la utilización de bienes o servicios subvencionados o gratuitos) para los empleados actuales;

(b) beneficios a los empleados retirados o jubilados, tales como beneficios por pensiones y otros beneficios por retiro, seguros de vida y atención médica para los retirados;

(c) otros beneficios a largo plazo, entre los que se incluyen las ausencias remuneradas después de largos periodos de servicio o sabáticas, los beneficios por acumulación de años de servicio y otros beneficios posteriores a un largo tiempo de servicio, los beneficios por incapacidad y, si no deben pagarse dentro de los doce meses del cierre del periodo, la participación en ganancias, incentivos y la compensación diferida; $y$

(d) beneficios por terminación.

Como podemos apreciar, la participación de los trabajadores en las utilidades se encuentra considerada dentro de los beneficios a los empleados a corto plazo y como tal le corresponden requerimientos de reconocimiento y medición específicos. 
Reconocimiento y medición de la participación de los trabajadores en las utilidades de la empresa

Se reconocerá el importe (sin descontar) de los beneficios a corto plazo:

(a) como un pasivo (gasto devengado), después de deducir cualquier pago a cuenta realizado. Si el importe pagado es superior al importe sin descontar de los beneficios, la entidad reconocerá la diferencia como un activo (pago anticipado de un gasto), en la medida que el pago por adelantado vaya a dar lugar, por ejemplo, a una reducción en los pagos a efectuar en el futuro o a un reembolso en efectivo; $y$

(b) como un gasto, a menos que otra Norma exija o permita la inclusión de los mencionados beneficios en el costo de un activo (por ejemplo en las existencias, según la NIC 2 Inventarios, y en los activos fijos, según la NIC 16 Propiedades, Planta y Equipo).

Es importante señalar que al aplicar lo señalado en (a) y (b) a la participación de los trabajadores en las utilidades de la empresa, una entidad reconocerá el costo esperado de esta participación cuando:

- Tenga una obligación presente, legal o implícita, de hacer tales pagos como consecuencia de sucesos ocurridos en el pasado; y

- Pueda realizarse una estimación fiable de la obligación.

En el caso de la participación de los trabajadores en las utilidades de la empresa, la NIC 19 señala que los empleados sólo recibirán una porción de las ganancias si permanecen en la entidad durante un periodo de tiempo especificado; sin embargo en nuestro país, la participación en las utilidades de los trabajadores de la empresa se distribuye en forma proporcional al tiempo en que el empleado prestó sus servicios a la empresa en un periodo determinado. Esta situación crea una obligación implícita a medida que los empleados prestan los servicios que incrementa el importe a pagar si permanecieran en servicio hasta el final del periodo especificado.

De lo señalado en la NIC 19 se desprende que una entidad podrá realizar una estimación fiable de la cuantía de sus obligaciones legales o implícitas como consecuencia de la participación de los trabajadores en las utilidades de la empresa, cuando:

(a) los términos formales de la participación contengan una fórmula para determinar el importe del beneficio;

(b) la entidad determine los importes a pagar antes de que los estados financieros sean autorizados para su emisión; o

(c) la experiencia pasada suministre evidencia clara acerca del importe de la obligación implícita por parte de la entidad.

\section{NORMATIVIDAD TRIBUTARIA DE LA PARTICIPACIÓN DE UTILIDADES.}

Para efectos tributarios, el artículo $\mathrm{N}^{\circ} 10$ del Decreto Legislativo No 892 establece que la participación legal de los trabajadores en las utilidades y las que el empleador otorgue unilateralmente o por convenio colectivo, constituyen gastos deducibles para determinar la renta imponible de la tercera categoría. En complemento de lo señalado, el inciso v) del artículo No $37^{\circ}$ de la Ley del Impuesto a la Renta (LIR) determina que son deducibles de la renta neta de la tercera categoría (empresas), los gastos o costos que constituyan para su perceptor rentas de quinta categoría, en el ejercicio gravable que les corresponda, cuando hayan sido pagadas dentro del plazo establecido por el Reglamento para la presentación de la Declaración Anual del Impuesto a la Renta de dicho ejercicio; o, 
en su caso, en el ejercicio gravable en que se paguen, aún cuando se encuentren debidamente provisionadas en un ejercicio anterior (cuadragésima octava disposición final de la LIR). El inciso c) del artículo No 34 de la LIR determina que la participación anual en las utilidades de las empresas constituye renta de quinta categoría para los trabajadores

En su pronunciamiento sobre el tema, la administración tributaria, a través del Informe No 033-2012-SUNAT/4B0000, concluye que, para la determinación de la renta imponible de la tercera categoría, la participación de los trabajadores en las utilidades de las empresas, en todos los casos, debe ser considerada - para efectos tributarios como un gasto deducible del ejercicio al que corresponda, siempre que se pague dentro del plazo previsto para la presentación de la declaración jurada anual del ejercicio (inciso v del artículo No 37 de la LIR).

$\mathrm{Al}$ respecto, la administración tributaria señala que, "aun cuando la participación de los trabajadores en las utilidades de las empresas podría ser reconocida como parte del costo de un activo, para la determinación del Impuesto a la Renta, ello no es posible, dado que existe una regulación tributaria específica que dispone que dicho concepto debe ser deducido como gasto".

El Informe bajo comentario ratifica dicha posición estableciendo que "como quiera que la legislación tributaria regula expresamente el tratamiento que se le debe otorgar a la participación en comentario, para efecto de la determinación del Impuesto a la Renta no resulta de aplicación la Resolución No 046-2011-EF/94 del Consejo Normativo de Contabilidad, más aún cuando dicha Resolución contiene disposiciones opuestas a las tributarias”. Según la posición de la administración tributaria, " no se podría cumplir con la exigencia dispuesta por el inciso v) del artículo No 37, habida cuenta que su reconocimiento estaría vinculado con la realización del activo y no con el pago de dicha participación a los trabajadores". Es importante señalar que, mediante nota al pie de página, que forma parte de su conclusión, la administración tributaria concluye en lo siguiente:

"Sin perjuicio de ello, el artículo $33^{\circ}$ del Reglamento de la Ley del Impuesto a la Renta dispone que la contabilización de operaciones bajo principios de contabilidad generalmente aceptados, puede determinar, por la aplicación de las normas contenidas en la Ley, diferencias temporales y permanentes en la determinación de la renta neta. En consecuencia, salvo que la Ley o el Reglamento condicione la deducción al registro contable, la forma de contabilización de las operaciones no originará la pérdida de una deducción.

Agrega que las diferencias temporales y permanentes obligarán al ajuste del resultado según los registros contables en la declaración jurada.

1. De acuerdo con lo establecido en los párrafos precedentes, para efectos tributarios, tratándose de empresas comercializadoras o de servicios, el tratamiento contable de la participación anual de los trabajadores en las utilidades dispuesto por la NIC19 Beneficios a los empleados, no genera ningún impacto tributario porque deberá ser contabilizada como gastos de administración o venta, según corresponda.

2. Complementando lo establecido por la NIC No 19, según la NIC № 2 - Existencias, tratándose de empresas productoras, 
para aquellos trabajadores de fábrica o planta cuya remuneración ha sido considerada como parte del costo de producción, su participación en las utilidades del ejercicio, deberá ser contabilizada como parte de dicho costo. Así las cosas, será aplicada a resultados y transferida al costo de ventas en la oportunidad en que los productos se vendan.

Desde un punto de vista contable, no cabe ninguna duda que parte de la participación de utilidades de los trabajadores - es decir, aquella que corresponde al personal de planta o fábrica, constituye costo de producción y, ulteriormente, costo de ventas, mientras que otra parte, -la vinculada a los trabajadores administrativos y de ventas - califica como gastos de administración o ventas, según corresponda. Los costos de transformación de los inventarios comprenden aquellos que se relacionan con las unidades producidas, tales como la mano de obra directa. Por otro lado, los costos indirectos fijos incluyen el referido a la gestión y administración de la planta y los costos indirectos variables a la mano de obra indirecta.

Cuando los inventarios sean vendidos, el importe registrado de los mismos, - al valor neto de adquisición o realización, el menor - se reconocerá como gasto del ejercicio en que se reconozcan sus correspondientes ingresos de operación. Así las cosas, el costo asume la categoría de gasto en el ejercicio en que se genere el beneficio para la empresa.

\section{APLICACIÓN DE LA PARTICIPACIÓN DE UTILIDADES A LOS COSTOS Y GASTOS}

El presente ensayo está referido a comentar específicamente el concepto utilidades considerado en los beneficios a corto plazo de la NIC 19, entendiéndose que anteriormente la misma estaba considerada en al NIC 12 donde, al comprender como parte de los beneficios del trabajador, afecta el concepto de costo y gasto y plantea problemas de medición y precios desde la doctrina de los costos

Comienzo por hacer un alcance de lo que se define como mano de obra:

La mano de obra o trabajo fabril representa el factor humano de la producción sin cuya intervención no podría realizarse la actividad manufacturera, independientemente del grado de desarrollo mecánico o automático de los procesos transformativos.

\section{CARACTERÍSTICAS DE LA MANO DE OBRA}

Entra algunas de las principales características de la mano de obra encontramos lo siguiente:

1. Pueden mejorar y perfeccionar el empleo y diseño de los recursos materiales y técnicos, lo cual no sucede a la inversa.

2. No pueden ser propiedad de la organización, a diferencia de los otros recursos. Los conocimientos, la experiencia, las habilidades, etc.; son parte del patrimonio personal.

3. Las actividades de las personas en las organizaciones son, como se apuntó, voluntarias; pero, no por el hecho de existir un contrato de trabajo la organización va a contar con el mejor esfuerzo de sus miembros; por lo contrario, solamente contará con él si perciben que esa actitud va a ser provechosa en alguna forma.

4. Las experiencias, los conocimientos, las habilidades, etc., intangibles, se manifiestan solamente a través del comportamiento de las personas en las organizaciones. Los miembros de ellas prestan un servicio a cam- 
bio de una remuneración económica y afectiva.

5. El total de recursos humanos de un país o de una organización en un momento dado puede ser incrementado. Básicamente existen dos formas para tal fin: descubrimiento y mejoramiento.

6. Los recursos humanos son variables de una persona a otra; no todo mundo posee las mismas habilidades, conocimientos, etc.

\section{IMPORTANCIA DE LA MANO DE OBRA}

Su importancia radica en que es el factor de producción por excelencia, debido a que es el que desarrolla una serie de actividades y tareas, y ayudado por instrumentos, infraestructura, entre otros, produce bienes y servicios de una manera satisfactoria.

\section{CLASIFICACIÓN}

La clasificación de la mano de obra va a depender directamente de la relación del trabajador con el proceso de fabricación del producto:

- Mano de obra directa: es la fuerza laboral que se encuentra en contacto directo con la fabricación de un determinado producto que tiene que producir la empresa. Esta ha sido responsable del grueso manejo del trabajo por horas de las fábricas y el costo más importante de controlar y medir.

- Mano de obra indirecta: es la fuerza labora que no se encuentra en contacto directo con el proceso de la fabricación de un determinado producto que tiene que producir la empresa. Entre los tipos de manos de obra tenemos: la recepción, oficinistas, servicios de limpieza, dibujantes, etc.
La señora de Roover en su fascinante estudio acerca de los registros contables del impresor de Amberes, Cristopher Plantin, sostiene que la primera aplicación de la contabilidad en la industria se efectuó a principios del siglo XIV en Italia. Contamos con una imagen bastante detallada de su desarrollo en los siguientes dos siglos gracias a su esposo, Raymond de Roover, quien nos proporcionó una relación de la organización de negocios establecida por los miembros de la familia Medici en Florencia durante el siglo XVI para la fabricación de lana. La compañía se funó en 1531. En esa fecha, según el estudio, en aquella sociedad solo es percibido como mano de obra el salario o jornal que se pagaba al trabajador, dado que los conceptos de derechos laborales surgen muchos años después.

Oscar Gómez Bravo en su libro Contabilidad de costos nos da una definición de la mano de obra:

El pago de los trabajadores y demás personas que laboran en una empresa constituye la mano de obra sea esta directa o indirecta.

Por otro lado John Neunner, uno de los clásicos de la teoría de costos, nos dice que la mano de obra son los costos por los servicios prestados y está integrada por la retribución monetaria que se da por el trabajo realizado, más los costos marginales del trabajo tal como los impuestos sobra la nómina.(1)

En esta línea de análisis diríamos que el considerar la NIC 19, la" participación en las ganancias" de la empresa (léase utilidades), trae como problema que para que se genere la misma debe ocurrir el hecho económico medible en un periodo contable, es decir primero debe ocurrir la producción y la posterior venta para que luego ocurra la generación de utilidades y la misma, de conformidad con las prácticas contables, se determine con precisión al término de cada año. 
Es decir que los productos elaborados hayan sido producidos y luego ser vendidos, por lo tanto, haber generado los costos y gastos necesarios para elaborarlos y distribuirlos en ese periodo. $\mathrm{Y}$ en consecuencia haber generado real y efectivamente la ganancia intrínseca en el precio de los mismos. Teniendo en cuenta que a diferencia de épocas anteriores los mercados hoy en día son cambiantes, el predeterminar ganancias por un trabajo realizado no siempre es factible y posible, más aun cuando están referidos organizaciones no corporativas.

Si bien es cierto que la definición de mano de obra deja abierta la posibilidad de considerar estos beneficios señalados en la NIC19 en los costos marginales de la misma, precísase que esté referida a hechos medibles con certeza.

EFECTOS EN LA ESTRUCTURA FINANCIERA Y LOS ANÁLISIS DE GESTIÓN.

En la formulación de los estados financieros se mostraban resultados después de la utilidad operativa y otros ingresos (egresos) al considerarlo como parte del costo de la mano de obra:

1. Se ha trasladado a los resultados operativos modificando el indicador de gestión de: RESULTADO OPERATIVO / VENTAS

2. Por la parte que afecta al costo de producción vendido modifica la utilidad bruta y el indicador de gestión:

\section{UTILIDAD BRUTA / VENTAS}

\begin{tabular}{|c|c|c|c|c|c|c|c|c|}
\hline & \multicolumn{4}{|c|}{ INTRADVECO EEFF2010 } & \multicolumn{4}{|c|}{ GOODYEAR EEFF2010 } \\
\hline \multirow{2}{*}{$\begin{array}{l}\text { ESTADO DE RESULTADOS } \\
\text { Ventas netas }\end{array}$} & \multicolumn{2}{|l|}{ EN 2011} & \multicolumn{2}{|l|}{ EN 2010} & \multicolumn{2}{|l|}{ EN 2011} & \multicolumn{2}{|l|}{ EN 2010} \\
\hline & 376,127 & $100 \%$ & \begin{tabular}{|l|l}
376,127 \\
\end{tabular} & $100 \%$ & 400,473 & $100 \%$ & 400,473 & $100 \%$ \\
\hline Costo de ventas & $(266,218)$ & $.71 \%$ & $(256,055)$ & $-68 \%$ & $(308,959)$ & $-77 \%$ & $(303,944)$ & $.76 \%$ \\
\hline Utilidad bruta & 109,909 & $29 \%$ & 120,072 & $32 \%$ & 91,514 & $23 \%$ & 96,529 & $24 \%$ \\
\hline Gastos de operación- neto & $(67,001)$ & $-18 \%$ & $(62,001)$ & $-16 \%$ & $(39,232)$ & $-10 \%$ & $(37,787)$ & $-9 \%$ \\
\hline Utilidad de operación & 42,908 & $11 \%$ & 58,071 & $15 \%$ & 52,282 & $13 \%$ & 58,742 & $15 \%$ \\
\hline Otros ingresos y gastos: & $-8,309$ & $-2 \%$ & $-8,310$ & $-2 \%$ & $\begin{array}{l}3,039 \\
\end{array}$ & $1 \%$ & $\begin{array}{l}3,039 \\
\end{array}$ & $1 \%$ \\
\hline Utilidad antes Partic. e Imp. & 34,599 & $9 \%$ & 49,761 & $13 \%$ & 55,321 & $14 \%$ & 61,781 & $15 \%$ \\
\hline Participación de utilidades & & $0 \%$ & $(4,977)$ & $-1 \%$ & & $0 \%$ & $(6,371)$ & $-2 \%$ \\
\hline npuesto a la Renta & $(10,196)$ & $-3 \%$ & $(13,437)$ & $-4 \%$ & $(17,179)$ & $-4 \%$ & $(17,206)$ & $-4 \%$ \\
\hline & 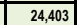 & $6 \%$ & 31,347 & 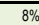 & ,142 & $10 \%$ & 38,204 & 10 \\
\hline
\end{tabular}

Los gerentes de la empresa deben de conocer y operar constantemente estos ratios para poder controlar la evolución de las ventas y la rentabilidad de su empresa. Muchas veces las políticas comerciales pueden derivarse en tres direcciones:

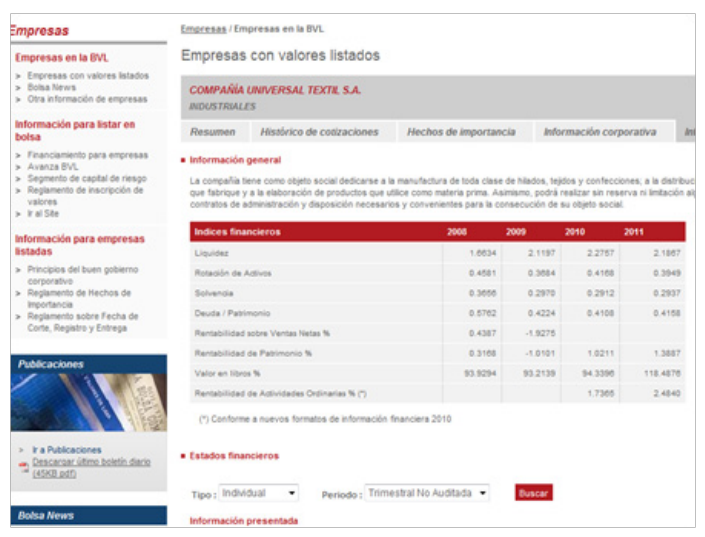

- Políticas orientadas a conseguir un alto valor del margen de la empresa, no interesa la cobertura comercial (volumen), sino el valor agregado y mantener el precio de mercado durante un periodo de tiempo.

- Políticas de volumen, cuando la empresa se orienta a coberturar el mercado masificando el crecimiento de la empresa en el mercado, esto puede en la mayoría de los casos sacrificio del margen, como contraresultado, el mercado "acepta" el precio y después resulta difícil intentar reposicionar el precio a un nivel mayor.

- Políticas equilibradas, donde la empresa diseña el plan de negocios, con márgenes adecuados comprometidos y desarrollo del mercado simultáneamente.

Finalmente, el Margen $\mathrm{Uo}=(\mathrm{Uo}) /$ Ventas es un indicador bastante fidedigno del rendimiento empresarial en un periodo dado, aunque no refleja el valor de la rentabilidad neta o real de la empresa, porque no considera otros gastos como deudas financieras, 
tributos y otros gastos, sin embargo muestra como evoluciona la gestión de la empresa por parte de los gerentes involucrados en el proceso comercial.

En los últimos años en nuestro país se ha observado que en la medición financiera de negocios $1 / 2 \%$ ya es un factor en la toma de decisiones, si comparamos por ejemplo que las inversiones de ahorro fluctúan alrededor de $4 \%$ anual ; que señalamos como éxitos fluctuaciones de crecimiento y rendimientos de inversión de mas de $1 \%$ y como vemos en los cambios de formulación las fluctuación pueden ser alrededor del $2 \%$.

No olvidemos que de aplicar lo expuesto en la norma, el valor de las existencias en el activo corriente del balance general se vería afectado.

Por otro lado, conociendo que nuestro Plan Contable General Empresarial (PCGE) está estructurado considerando el reconocimiento de los hechos económicos sobre la base de las NORMAS INTERNACIONALES DE INFORMACIÓN FINANCIERA se tendría que incorporar en los códigos de cuentas las que representen la participación de utilidades como beneficios sociales del trabajador y modificar la estructura de las cuentas de saldos intermedios de gestión.

Es importante mencionar que se debe definir las políticas para el tratamiento de costos inventariables con inclusión de la NIC 19 en periodos intermedios.

\section{GASTOS DE PERSONAL, DIRECTO- RES Y GERENTES}

621 Remuneraciones

622 Otras remuneraciones

623 Indemnizaciones al personal

624 Capacitación
625 Atención al personal

626 Gerentes

627 Seguridad, previsión social y otras

628 Retribuciones al directorio

629 Beneficios sociales de los trabajadores

6291 Compensación por tiempo de servicio

6292 Pensiones y jubilaciones

6293 Otros beneficios posempleo.

87 PARTICIPACIONES DE LOS TRABAJADORES

871 Participación de los trabajadores - Corriente

872 Participación de los trabajadores - Diferida

\section{CONCLUSIÓN}

Del análisis de la Norma Internacional de Contabilidad - NIC 19.

Podemos concluir que las obligaciones relacionadas con la participación de los trabajadores en las utilidades de la empresa son consecuencia de los servicios prestados por los empleados, no de transacciones con los propietarios. Por tanto, la entidad reconocerá el costo de las participaciones como un gasto y no como un componente de la distribución de la ganancia.

Cabe señalar que los gastos pueden clasificarse en gastos operativos, costo de ventas y otros gastos y que, cuando la NIC 19 indica que se reconocerá el costo de las participaciones de los trabajadores en las utilidades de la empresa como un gasto, éstas podrán ser reconocidas como gasto operativo o como costo de ventas.

Por otro lado, el reconocer en el gasto por la participación de los trabajadores en las utilidades de la empresa implica a su vez reconocer el importe de esta participación como un pasivo para la empresa. 


\section{Del análisis tributario.}

Tomando en consideración lo señalado por el artículo No 33 del Reglamento de la LIR, el tratamiento contable de la participación de los trabajadores de fábrica en las utilidades del ejercicio en que se generan, determina una diferencia temporal para efectos de la determinación de la renta neta imponible. $\mathrm{Al}$ ser reconocida como gasto del ejercicio para efectos del Impuesto a la Renta, se genera un impuesto a la renta diferido pasivo, que deberá ser compensado en el ejercicio en que se produzca la venta de los bienes y, consecuentemente, se materialice la transferencia del costo de producción al costo de ventas. Según lo establecido por el inciso v) del artículo No 37 de la LIR, el reconocimiento tributario del gasto será efectuado vía Declaración Jurada anual del Impuesto a la Renta del ejercicio en el que efectivamente se pague.

Efecto de la aplicación de la NIC No 19 en el tratamiento tributario de los precios de transferencia.

La legislación peruana de "precios de transferencia" referida a operaciones entre partes vinculadas, a la que se refiere el numeral 4 del artículo No 32 y el artículo No $32 \mathrm{~A}$ de la LIR y normas modificatorias, así como su Reglamento aprobado mediante Decreto Supremo No 190-2005-EF, incorpora la utilización de seis métodos de validación de los valores de mercado aplicables, así como la posibilidad de efectuar ajustes en la determinación de los precios de transferencia para efectos tributarios.

En los tres primeros métodos de validación, denominados directos, los comparables son los precios de los productos o servicios, $\mathrm{y}$, en los tres últimos métodos denominados indirectos, lo que se comparan son las fun- ciones de las empresas, incorporando la información financiera correspondiente. La aplicación de los mencionados métodos puede determinar ajustes en la determinación de los precios de transferencia para efectos tributarios.

Los métodos indirectos, en particular, el "método del margen neto transaccional", se caracterizan por comparar los beneficios de las transacciones entre partes vinculadas, utilizando indicadores financieros - ratios de rentabilidad -, tales como "utilidad de operación sobre ventas".

Los ratios de rentabilidad surgen de la información financiera de la empresa y de las empresas comparables, específicamente del "Estado de Resultados". Así las cosas, el mencionado "estado" estará influenciado por la aplicación de la NIC19 para el tratamiento contable de la participación de los trabajadores en las utilidades. Es así que, cuando se utilice en un estudio técnico de precios de Transferencia" el método del "margen neto transaccional", la determinación de la utilidad de operación estará disminuida habida cuenta de que la participación de los trabajadores a pagar, será considerada como costo o gasto del ejercicio.

Tomando en cuenta la aplicación del método estadístico denominado "intercuartil", lo mencionado en los párrafos precedentes, puede generar que los valores de mercado de las operaciones entre partes vinculadas se sitúen fuera del "rango de precios" en el que se obtiene el cálculo de la "mediana", con la consecuente determinación de ajustes tributarios.

Si la utilidad BASE IMPONIBLE para la determinación de la participación deter- 
mina que dicha participación es deducible si se paga dentro del plazo, debería precisarse si la participación asignada a los costos que se presenta en los inventarios son deducibles para determinar el impuesto a la renta.

\section{Del análisis de costos}

Problema de medición al considerar la NIC 19 como beneficios del trabajador. Sin embargo debe entenderse que está referida a ser considerada como un costo marginal del trabajo. Al hacerlo así genera específicamente un problema de medición de costos de producción de los objetivos del costo (productos y servicios). Colisionando de esta manera con el concepto de costos de producción que indica que son todos los esfuerzos medibles incurridos necesariamente (concepto económico) en la producción o prestación de servicios en un periodo determinado

Según el marco conceptual de las Normas Contables se habla de reconocer un gasto cuando el mismo sirve para la obtención específica de ingresos. Uno de los problemas es cómo incluir al cierre del año utilidades que puedan ser determinadas según el DL 892(norma local). Debe recordarse que los costos de producción estan regidos principalmente por el principio de devengado.

Finalmente como el enfoque que mayormente siguen las NIC's son básicamente financieros, muchas de las veces no consideran los conceptos que son comprendidos en la Doctrina de los Costos.

La aplicación de la citada norma genera, en las empresas formales obligadas a aplicarlas de manera obligatoria desde el 2011, problemas de valuación de inventarios y registro y se contrapone al concepto de medición del costo
Es importante definir y determinar la estructura de costos que permita asociar la participación a dichos costos:

Fábrica de Muebles y Cabinas de Internet
Estado de costos de producción
Par la distribución de la participación de utilidades 2,012
(en nuevos soles)
\begin{tabular}{|l|r|r|r|r|r|}
\hline CATEGORIAS DE COSTOS & DE MES & \multicolumn{1}{l|}{ ACUMLLADU } \\
\hline MATERIALES & 254,400 & $69.1 \%$ & $3,052,800$ & $70.2 \%$ \\
\hline MANO DE OBRA DIRECTA & 38,160 & $10.4 \%$ & 457,920 & $10.5 \%$ \\
\hline MANO DE POBRA INDIRECTA & 12,720 & $3.5 \%$ & 152,640 & $3.5 \%$ \\
\hline SERVICIOS CON PARTIC.DE UTLIDADES & 18,000 & $4.9 \%$ & 200,000 & $4.6 \%$ \\
\hline SERVICIOS & & 25,000 & $6.8 \%$ & 300,000 & $6.9 \%$ \\
\hline COSTO INDIRECTOS DEPRODUCCION & 20,000 & $5.4 \%$ & 183,168 & $4.2 \%$ \\
\hline TOTAL & & 368,280 & $100.0 \%$ & $4,346,528$ & $100.0 \%$ \\
\hline
\end{tabular}

\section{Del análisis financieros}

$\mathrm{Al}$ incorporar las participaciones de trabajadores como costos y gastos existe el riesgo de modificar la base que usualmente sirve para formular políticas de precios o márgenes de utilidad BRUTA Y/O OPERATIVA.

Cuando se efectúan análisis financieros sobre la base de flujos futuros se debe tomar en cuenta el cambio de la estructura de los resultados de UTILIDADES BRUTAS Y OPERATIVAS.

Se debe integrar al PCGE el código de cuenta que incluya los BENEFICIOS DE PARTICIPACIÓN DE UTILIDADES.

Se debe eliminara del PCGE la cuenta:

ELEMENTO 8: SALDOS INTERMEDIARIOS DE GESTIÓN Y DETERMINACIÓN DEL RESULTADO DEL EJERCICIO

87 PARTICIPACIONES DE LOS TRABAJADORES.

En una siguiente publicación expondremos los análisis necesarios para la aplicación de esta norma al costeo del producto, determinación del costo inventariable y su tratamiento para la formulación de estados financieros en PERIODOS INTERMEDIOS. 


\section{PROPUESTA}

a) Para ACELERAR el cumplimiento de la norma la gerencia debe formular políticas para el tratamiento de distribución de la participación a costos y gastos, incluyendo los procesos de periodicidad, registros, responsables de cumplimiento.

b) Para SIMPLIFICAR el proceso en contador debe definir la estructura del sistema de información para la segmentación de la participación de utilidades a las áreas respectivas e identificar la relación periodo- proceso- producto al que estaría asociado dicha participación.

b) Para ASEGURAR el proceso de cumplimientos, el contador debe documentar la política; proponemos el siguiente modelo:

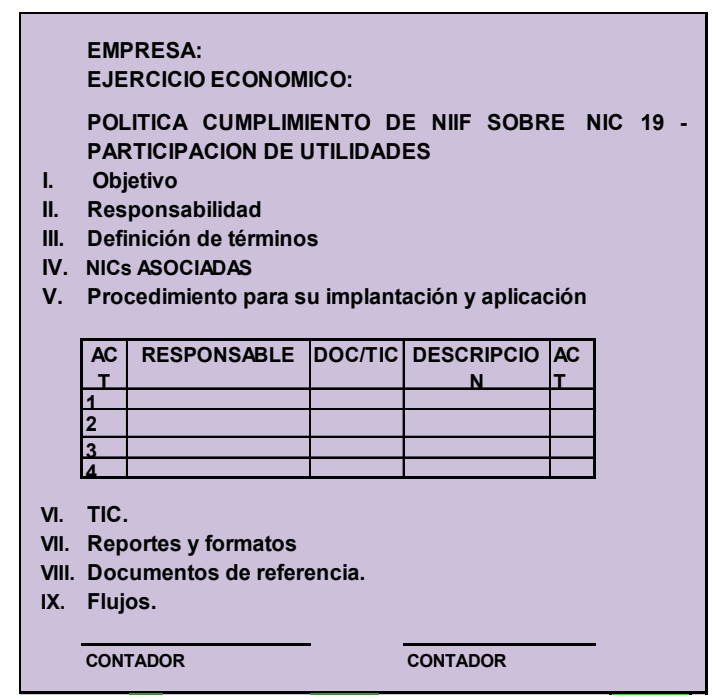

d) Las normas laborales y tributarias deben recoger el reconocimiento de la participación de utilidades como beneficios del trabajador y precisar su tratamiento como costo computable y obligaciones de su oportunidad de pago. e) Precisar en las normas tributarias laborales si al ser considerado beneficio del trabajador y recibirlo en más de un periodo, estaría afecto a los impuestos y contribuciones ( además de la retención de impuesto a la renta de quinta categoría).

f) Un modelo de distribución de costos podría ser efectuando la distribución de la participación primero entre los costos de distribución (gastos operativos) del periodo. Para esto es necesario determinar la participación de la mano de obra directa e indirecta de la mano de obra en la producción.

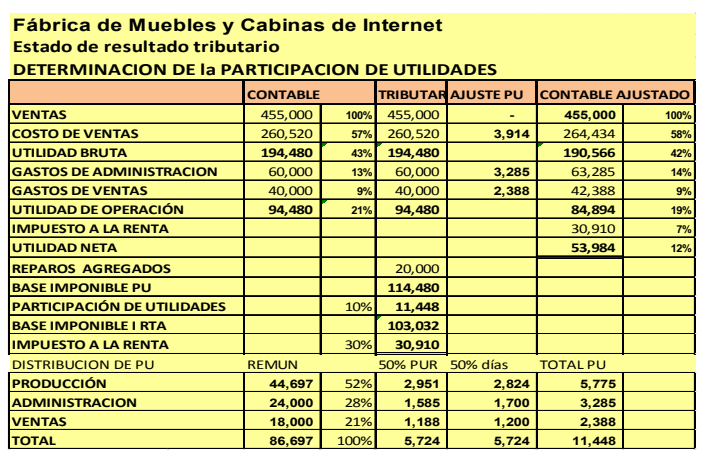

Luego distribuir la participación de utilidades, asignada a la producción, a los costos de ventas e inventarios finales de productos terminados e inventarios en procesos

\begin{tabular}{|c|c|c|c|c|c|c|}
\hline \multicolumn{7}{|c|}{$\begin{array}{l}\text { Fábrica de Muebles y Cabinas de Internet } \\
\text { Estado de costo de producción ejercicio } 2011\end{array}$} \\
\hline & CTOUNIT PR & TOTAL & INVPT & INV PTERM & INV PPROC & CTO VTA \\
\hline Volumen & & 2000 & 500 & 500 & 200 & 1300 \\
\hline MATERIALES & 153.00 & 305,280 & $77 \%$ & 76,500 & 29,880 & 198,900 \\
\hline$M . O . D+1$ & 34.25 & 65,704 & $17 \%$ & 17,125 & 4,054 & 44,525 \\
\hline CIP & 13.15 & 25,122 & $6 \%$ & 6,575 & 1,452 & 17,095 \\
\hline TOTAL & 200.40 & 396,106 & $100 \%$ & 100,200 & 35,386 & 260,520 \\
\hline DISTRIB PU & & 5,775 & & 1,505 & 356 & 3,914 \\
\hline \multicolumn{2}{|c|}{ COSTO AJUSTADO POR PU } & 401,881 & & 101,705 & 35,742 & 264,434 \\
\hline \multicolumn{2}{|l|}{ M.O.D+I } & 65,704 & & 17,125 & 4,054 & 44,525 \\
\hline \multicolumn{2}{|c|}{\begin{tabular}{|l|} 
PARTICIPACION DE UTILIDADES \\
\end{tabular}} & 5,775 & $8.79 \%$ & 1,505 & 356 & 3,914 \\
\hline
\end{tabular}

g) Para los ejercicios 2010, 2011, 2012 se debe incluir una nota a los estados financieros para exponer el cambio en los indicado- 
res de gestión comparando los ratios financieros con la aplicación de la norma y sin ella.

h) Modificar el PLAN CONTABLE GENERAL EMPRESARIAL INCLUYENDO: ELEMENTO 6: GASTOS POR NATURALEZA

62 GASTOS DE PERSONAL, DIRECTORES Y GERENTES

629 Beneficios sociales de los trabajadores 6291 Compensación por tiempo de servicio 6292 Pensiones y jubilaciones 6293 Otros beneficios posempleo 6294 Participación de utilidades

\section{REFERENCIAS BIBLIOGRÁFICAS:}

1. IFRS NIC 19 "Beneficios a empleados, vigencia para los estados financieros que cubran periodos que comiencen a partir del 1 de enero de 1999”.

2. RESOLUCIÓN No 046-2011EF/94, Consejo Normativo de Contabilidad, del Lima, 27 de enero de 2011.
3. HORNGREN, CHARLES T, Edición (2006). Contabilidad de costos un enfoque gerencial. México D.F. México.

4. GOMEZ BRAVO, Oscar. (2000). Contabilidad de costos. Mc. Graw Hill.

5. Neuner-Deakin. Contabilidad de costos. Tomo 1. LIMUSA.

6. Decreto Legislativo No 892, Artículo $10^{\circ}$ publicado el 11.11 .1996 y normas modificatorias.

7. Texto Único Ordenado de la Ley del Impuesto a la Renta, aprobado por el Decreto Supremo N. ${ }^{\circ}$ 179-2004-EF, publicado el 8.12. 2004, y normas modificatorias (en adelante, TUO de la Ley del Impuesto a la Renta).

8. InformeNo 033-2012-SUNAT/ 4B0000 del 13.04.12. 